\title{
Insulin analogues: too much noise about small benefits
}

\author{
Andrea Siebenhofer-Kroitzsch MD, Karl Horvath MD, Johannes Plank MD
}

$\infty \infty$

See related research paper by Singh and colleagues, page 385

$\mathrm{T}$ he development of insulins has long been central to improvements in the management of diabetes mellitus. The first insulin preparations varied significantly in potency. Also, contamination of insulin solutions was common. Thus, considerable attention was devoted to the development of higher-quality formulations. Intermediateand long-acting insulins were designed to establish stable background levels of insulin. Improved purification methods and recombinant production techniques virtually eliminated insulin dystrophy and allergy. With the strategy of intensive insulin therapy, a nearly physiologic model of insulin replacement has been introduced.

Nevertheless, poor glycemic control still occurs in many patients. ${ }^{1,2}$ This outcome is due in part to the essential limitation of using subcutaneous injections to deliver insulin systemically. However, it has also been attributed to insulin formulations that remain suboptimal. The introduction of rapid- and long-acting insulin analogues with action profiles resembling physiologic conditions more closely than conventional insulins yielded hope for substantial improvement in glycemic control.

In this issue of $C M A J$, Singh and colleagues ${ }^{3}$ report on the differences in glycemic control and risk of hypoglycemia between insulin analogues and conventional insulins in adults and children with type 1 diabetes, in adults with type 2 diabetes and in pregnant women with type 1 or gestational diabetes. Regrettably, their results again confirm that conclusions cannot be drawn about the long-term effects of insulin analogues on the risk of diabetes-related complications and death. Despite their efforts to include studies of at least 4 weeks' duration, only short-term trials were available. Singh and colleagues indicate that differences in hemoglobin $\mathrm{A}_{1 \mathrm{c}}$, risk of hypoglycemia and quality of life were at best minor and of clinically debatable relevance. Their findings are consistent with those of other systematic reviews and meta-analyses of insulin analogues. ${ }^{4-6}$

Singh and colleagues reported only on insulins that are marketed in Canada. A Cochrane review of rapid-acting insulin analogues published in 2006 looked at a third analogue, insulin glulisine. ${ }^{4}$ This rapid-acting analogue was shown to be no better than regular insulin in terms of glycemic control or reduced risk of hypoglycemia in patients with type 1 diabetes. Since then, another study that met the Cochrane criteria ${ }^{4}$ compared insulin glulisine and regular insulin in type 2 diabetes; it also showed no difference in effectiveness between the 2 insulins.

Despite the evidence, myths abound regarding the benefits

\section{Key points}

- The improved glycemic control, reduced risk of hypoglycemia and improved quality of life achieved with insulin analogues versus conventional insulins are at best minor and of clinically debatable relevance.

- Insulin analogues should be reserved for use in selected patients, such as those with nocturnal hypoglycemia.

- Efforts should be focused on offering structured educational programs to help patients manage their diabetes and improve glycemic control.

of insulin analogues and require correction. First, compared with regular insulin, insulin analogues produce only minor additional reductions in hemoglobin $\mathrm{A}_{\mathrm{lc}}$. For all such comparisons, whether of long-acting or rapid-acting insulin analogues in type 1 or type 2 diabetes, the observed difference in hemoglobin $\mathrm{A}_{\mathrm{lc}}$ is well below $0.4 \%$. This level is commonly accepted in studies as a margin of inferiority. Even if one assumed that reductions in hemoglobin $\mathrm{A}_{\mathrm{lc}}$ with insulin analogues would result in similar clinical benefits as those observed in the Diabetes Control and Complications Trial, ${ }^{8}$ about 650 patients with type 1 diabetes would have to be treated with rapid-acting insulin analogues for 1 year to prevent the development of retinopathy in 1 patient. $^{4}$

Second, severe episodes of hypoglycemia are rare. Even if nocturnal hypoglycemia occurs slightly less frequently with insulin analogues than with conventional insulins, the overall difference in hypoglycemia is small. A cross-sectional study in Germany involving 35723 patients (5891 with type 1 diabetes and 29693 with type 2 diabetes), 59\% of whom used insulin analogues, showed that treatment with insulin analogues was not associated with a decreased rate of severe hypoglycemia. ${ }^{9}$

Third, advantages in terms of quality of life reported with rapid-acting insulin analogues were driven mostly by changes in convenience, flexibility and adherence to treatment. Most of the studies had an open-label design. Patients were advised to inject regular insulin on average 30 minutes before meal time, whereas insulin analogues were injected immediately before meals. One may hypothesize that the difference in in-

All of the authors are with the Department of Internal Medicine, and Andrea Siebenhofer-Kroitzsch and Karl Horvath are with the EBM Review Center, Medical University of Graz, Graz, Austria. 
jection times is a major underlying reason for improved satisfaction with insulin analogues. The rationale for different injection times in intensified insulin therapy has never been proven in controlled studies. In daily life, most patients seem to use short injection-meal intervals or none at all. ${ }^{10}$ In addition, a double-blind trial comparing rapid-acting insulin analogues with regular insulin did not show an improvement in any quality-of-life measures with insulin analogues. ${ }^{11}$

The minor benefits seen with insulin analogues appear to be limited mainly to open-label study designs. A switch to a new insulin preparation, which may be strongly encouraged by physicians, consumer groups and industry, generally brings about an increase in patient motivation and compliance with other important aspects of diabetes care. Based on the available evidence in the report by Singh and colleagues and other systematic reviews, older conventional insulins remain effective. Therefore, the extensive promotion of insulin analogues is not justified. Insulin analogues should be reserved for use in selected patients, such as those with nocturnal hypoglycemia.

Structured programs for self-management training of patients with type 1 and type 2 diabetes have shown a more pronounced, clinically relevant and sustained improvement in glycemic control and reduction in risk of severe hypoglycemia $^{12-15}$ compared with the relative benefit observed to date with insulin analogues. Until well-designed long-term studies consistently demonstrate the benefits of insulin analogues, money should be spent on structured educational programs to improve outcomes for patients with diabetes.

Competing interests: The authors are part of a research group that has performed several studies of rapid- and long-acting insulin analogues with the companies Sanofi-Aventis, Eli Lilly and Novo Nordisk. They were involved in the preparation of a report on the effects of rapid- and long-acting insulin analogues for the German Institute for Quality and Efficiency in Health Care.

Contributors: All of the authors were involved in the preparation of this manuscript and approved the final version submitted for publication.

\section{REFERENCES}

1. Holman RR, Paul SK, Bethel MA, et al. 10-year follow-up of intensive glucose control in type 2 diabetes. N Engl J Med 2008;359:1577-89.

2. Nathan DM, Cleary PA, Backlund JY, et al. Intensive diabetes treatment and cardiovascular disease in patients with type 1 diabetes. N Engl J Med 2005;353:2643-53.

3. Singh SR, Ahmad F, Lal A, et al. Efficacy and safety of insulin analogues for the management of diabetes mellitus: a meta-analysis. CMAJ 2009;180:385-97.

4. Siebenhofer A, Plank J, Berghold A, et al. Short acting insulin analogues versus regular human insulin in patients with diabetes mellitus. Cochrane Database Syst Rev 2006;(2):CD003287.

5. Horvath K, Jeitler K, Berghold A, et al. Long-acting insulin analogues versus NPH insulin (human isophane insulin) for type 2 diabetes mellitus. Cochrane Database Syst Rev 2007;(2):CD005613.

6. Rapid-acting insulin analogues in the treatment of diabetes mellitus type 1. Cologne (Germany): Institute for Quality and Efficiency in Health Care; 2005. Available: www .iqwig.de/download/A05-02_Executive_Summary_Rapid-acting_insulin_analogues _in_the_treatment_of_diabetes_mellitus_type_1.pdf (accessed 2009 Jan. 7).

7. Rayman G, Profozic V, Middle M. Insulin glulisine imparts effective glycaemic control in patients with type 2 diabetes. Diabetes Res Clin Pract 2007;76:304-12.

8. Diabetes Control and Complications Trial Research Group. The effect of intensive treatment of diabetes on the development and progression of long-term complications in insulin-dependent diabetes mellitus. N Engl J Med 1993;329:977-86.

9. Müller N, Schiel R, Osterbrink B, et al. Care and outcome in patients with diabetes mellitus type 1 and type 2 in specialized practice, diabetes specialized hospital and diabetes department of a regional general hospital in Germany 2005. Diabetologie Stoffwechsel 2008;3:41-50.

10. Heinemann L. Do insulin-treated diabetic patients use an injection-meal-interval in daily life? Diabet Med 1995;12:449-50.

11. Gale EA. A randomized, controlled trial comparing insulin lispro with human soluble insulin in patients with type 1 diabetes on intensified insulin therapy. The UK Trial Group. Diabet Med 2000;17:209-14.

12. DAFNE Study Group. Training in flexible, intensive insulin management to enable dietary freedom in people with type 1 diabetes: dose adjustment for normal eating (DAFNE) randomised controlled trial. BMJ 2002;325:746.

13. Pieber TR, Holler A, Siebenhofer A, et al. Evaluation of a structured teaching and treatment programme for type 2 diabetes in general practice in a rural area of Austria. Diabet Med 1995;12:349-54.

14. Plank J, Kohler G, Rakovac I, et al. Long-term evaluation of a structured outpatient education programme for intensified insulin therapy in patients with type 1 diabetes: a 12-year follow-up. Diabetologia 2004;47:1370-5.

15. Sämann A, Mühlhauser I, Bender R, et al. Glycaemic control and severe hypoglycaemia following training in flexible, intensive insulin therapy to enable dietary freedom in people with type 1 diabetes: a prospective implementation study. Diabetologia 2005;48:1965-70.

Correspondence to: Dr. Johannes Plank, Department of

Internal Medicine, Medical University of Graz,

Auenbruggerplatz 15, 8036 Graz, Austria; fax 43316385 4332;

johannes.plank@klinikum-graz.ator plank@diabetesambulanz.at

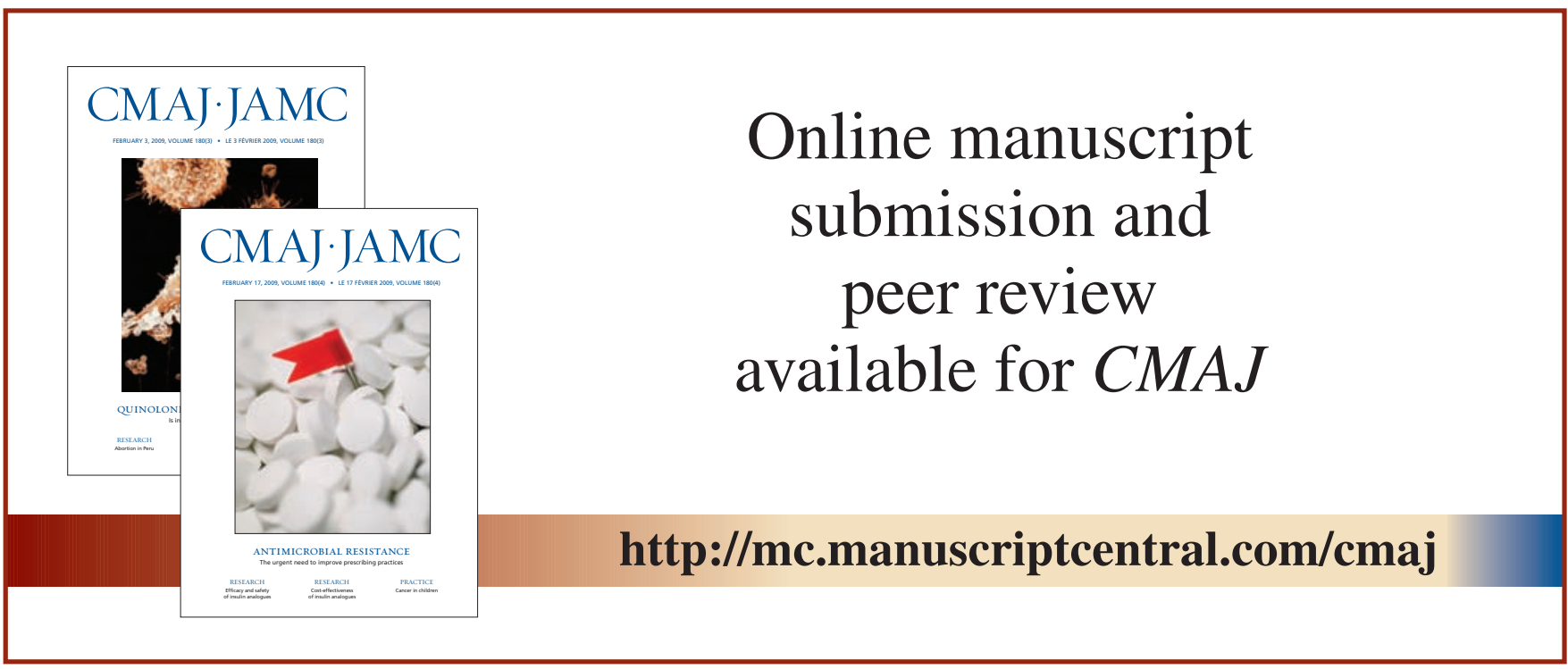

